\title{
Les âges du consentement. Militantisme gai et sexualité des mineurs en France et au Québec (1970-1980)
}

The age(s) of consent: gay activism and the sexuality of minors in France and Quebec (1970-1980)

Jean Bérard et Nicolas Sallée

\section{(2) OpenEdition Journals}

\section{Édition électronique}

URL : http://journals.openedition.org/clio/12778

DOI : 10.4000/clio. 12778

ISSN : $1777-5299$

\section{Éditeur}

Belin

Édition imprimée

Date de publication : 1 décembre 2015

Pagination : 99-124

ISBN : 9782701194325

ISSN : $1252-7017$

\section{Référence électronique}

Jean Bérard et Nicolas Sallée, « Les âges du consentement. Militantisme gai et sexualité des mineurs en France et au Québec (1970-1980) », Clio. Femmes, Genre, Histoire [En ligne], 42 | 2015, mis en ligne le 01 décembre 2018, consulté le 21 décembre 2020. URL : http://journals.openedition.org/clio/12778 ; DOI : https://doi.org/10.4000/clio.12778 


\title{
Les âges du consentement. Militantisme gai et sexualité des mineurs en France et au Québec (1970-1980)
}

\author{
Jean BÉRARD \& Nicolas SALLÉE
}

En France et au Québec, comme dans de nombreux autres pays, les «années 68 » ont été marquées par l'essor de nombreux mouvements contestataires, aux causes et aux revendications diversifiées ${ }^{1}$. Dans leur majorité, ces mouvements ont été portés par une jeunesse refusant les normes constitutives des modalités dominantes et autoritaires de régulation des conduites dans les institutions familiales, productives et répressives. Ces mouvements jugeaient « insupportables » la minorisation d'individus qui, renvoyés à leur âge, leur classe, leur sexe, leur genre ou leur orientation sexuelle, leur origine supposée ou leur statut assigné (élèves, prisonniers, malades, etc.), étaient soumis, légalement ou non, à diverses formes de disciplines corporelles et de privations ordinaires ${ }^{2}$. Quand cette minorisation était liée à l'âge, sa dénonciation portait sur la définition légale des seuils d'accès à différentes majorités, de la majorité civile, de fait liée à la majorité matrimoniale et à la majorité électorale, à la majorité sexuelle, fixant l'âge à partir duquel des personnes civilement majeures peuvent entretenir des relations sexuelles avec des personnes civilement mineures. L'enjeu politique lié à la majorité sexuelle était lié à un autre front de lutte des "années 68 »: en effet, à cette époque, il n'existait pas une mais des majorités sexuelles, dont la définition était étroitement liée à la répression de l'homosexualité.

1 Sur la France, voir par exemple Artières \& Zancarini-Fournel 2008; sur le Québec, Warren 2008.

2 Sur le déplacement des frontières du «supportable » et de «l'insupportable » dans les « années 68 », nous renvoyons aux travaux de Dominique Memmi (2008). 
En France, une ordonnance du 2 juillet 1945 fixait la majorité sexuelle générale à 15 ans. Reprenant un décret adopté par Vichy en 1942, le Code pénal sanctionnait à son article 331-3 (devenu 331-2 en 1980 , jusqu'à son abrogation en 1982) toute « relation sexuelle contrenature » avec un mineur ${ }^{3}$ (de moins de 21 ans jusqu'en 1974, 18 ans ensuite). Au Canada, et donc au Québec, la fixation des majorités sexuelles, liée au sexe et aux pratiques sexuelles, portait sur deux principes distincts qui, formulés plus explicitement qu'en France, étaient censés protéger l'entrée dans le mariage et la famille: la chasteté des jeunes filles contre des séductions trop précoces, et la moralité des jeunes garçons contre la «perversion» homosexuelle. Ainsi, le Code criminel de 1892 fixait à 14 ans un âge de la majorité sexuelle, alors réservé aux jeunes filles, et définissait - héritage du droit anglais - un crime de sodomie. Le bill Omnibus de 1969 a certes ouvert le droit à la sodomie, ainsi d'ailleurs qu'aux actes dits de " grossière indécence » ${ }^{4}$, mais son article 158 les réservait "à un mari et sa femme», ainsi qu' «à deux personnes consentantes âgées de 21 ans ou plus $»^{5}$. Si une réforme - fédérale - de 1988 a abaissé cet âge à 18 ans, il a fallu attendre une décision de justice - provinciale pour qu'au Québec, soit abrogé ce que l'on appellera désormais le « double standard» en matière de majorité sexuelle. En revenant sur l'histoire des mouvements gais français et québécois, cet article propose d'analyser la constitution, dans les années 1970, d'un espace politique de mise en discussion de la majorité sexuelle et de ses liens avec l'orientation sexuelle.

3 Marchant $2006: 180$.

4 Les actes de « grossière indécence » étaient définis comme des actes sexuels, sans assaut (pour les distinguer de l'attentat à la pudeur) et sans pénétration (pour les distinguer de la sodomie), qui contrevenaient à la moralité. Jusque 1955, cette qualification était légalement réservée aux homosexuels masculins.

5 À cette époque, au Québec, la majorité civile était de 21 ans, jusqu’à une réforme de 1971 qui l'a abaissée à 18 ans. Contrairement à la définition française, cet abaissement de la majorité civile n'a pas eu de conséquence directe sur les seuils légaux de majorité sexuelle. Si en effet, la fixation du seuil de la majorité civile est de compétence provinciale, la fixation des seuils de la majorité sexuelle est, quant à elle, de compétence fédérale. 
Au début des années 1970, les mouvements gais héritent donc d'un enjeu légal comparable, en France et au Québec: l'âge de la majorité sexuelle, plus élevé pour les homosexuels, soulève la question de la liberté sexuelle des jeunes mineurs, voire des enfants et des jeunes adolescents ${ }^{6}$. À ce moment, la fixation des majorités sexuelles n'est cependant pas la seule forme de répression de l'homosexualité. Tandis qu'en France, l'article 330-2 du Code pénal réprime «l'outrage public à la pudeur », pour lequel la peine minimale a été doublée, en 1960, en cas de rapports homosexuels, le Code criminel canadien réprime l'attentat à la pudeur d'un homme envers "une autre personne du sexe masculin». Un trait commun à ces infractions est qu'elles sont peu définies, ouvrant de larges possibilités d'arrestations et de poursuite contre les homosexuels, en particulier dans les bars et les lieux publics. En outre, si la répression de ces infractions varie selon les moments, elle s'abat toujours de manière plus importante en cas de relations entre un adulte et un mineur?

Ces lois font partie d'un ensemble normatif social et médical plus large, constituant l'homosexualité comme une «perversion » dont les enfants et les jeunes mineurs doivent être protégés, plus que de tout autre contact sexuel 8 . Dans ce cadre, la mise en question de l'écart d'âge dans l'accès aux sexualités porte une critique de la perception de l'homosexualité comme une menace pour la jeunesse. Elle s'inscrit dans un mouvement plus large visant la normalisation de l'homosexualité. Dans le même temps, la politisation du thème de la majorité sexuelle a conduit à des positions controversées : se refusant à limiter leur revendication à la liberté sexuelle des jeunes mineurs, et

6 Ces différentes catégories, fluctuantes dans le temps, étaient en voie de cristallisation dans ces années d'après-guerre où les transitions vers l'âge adulte s'allongeaient progressivement. Dans cet article, nous réserverons le terme de «jeunes mineurs » aux mineurs de plus de 15 ans, celui de «jeunes adolescents » aux mineurs de 12 à 15 ans, et celui « d'enfants » aux mineurs de moins de 12 ans. Ces seuils, relativement arbitraires, ne doivent cependant pas être essentialisés.

7 Hurteau 1993 ; Tamagne 2000 ; Corriveau 2006.

8 Dans Les Anormaux, Michel Foucault souligne qu'à partir de la seconde moitié du $\mathrm{XIX}^{\mathrm{e}}$ siècle, l'enfance, durant laquelle les instincts sexuels peuvent être régulés, ou déborder vers l'anormalité, est «une des conditions historiques de la généralisation du savoir et du pouvoir psychiatriques » (Foucault 1999 : 297). 
à l'abrogation du double standard en matière de majorité sexuelle, certains militants ont revendiqué la liberté sexuelle des enfants et des jeunes adolescents, allant jusqu'à mettre en question l'existence même d'une majorité sexuelle?.

\section{Sources}

L'article s'appuie sur deux corpus de sources qui posent, tout d'abord, une question d'échelle de comparaison. Le premier corpus est constitué par des sources militantes, principalement les revues militantes gaies des années 1970 et du début des années 1980 et, secondairement, les livres publiés par des militants. Ces corpus permettent de comparer les positions militantes exprimées en France et au Québec ${ }^{10}$. Notre second corpus est constitué par les sources gouvernementales et législatives produites autour des réformes de la majorité sexuelle dans les années 1980. La loi pénale étant cependant, au Canada, une prérogative fédérale, l'article propose des va-et-vient entre les échelles québécoises et canadiennes, observant, en particulier, la manière dont les mouvements gais québécois se sont positionnés face aux évolutions législatives fédérales. Pourquoi, dans ce cadre, nous être limités au Québec ? Il y a au moins deux raisons à cela. La première, pragmatique, tient à la nécessité de circonscrire l'investigation. La seconde tient à la position singulière du militantisme gai québécois, et plus spécifiquement montréalais, dont l'histoire est liée non seulement à celle des mouvements canadiens anglophones, mais également à la circulation d'idées - facilitée par la proximité linguistique - entre la France et le Québec ${ }^{11}$.

La question de l'homosexualité féminine et des mouvements lesbiens constitue un point aveugle de notre travail. Cette limite est non seulement liée à la nature de notre premier corpus (archives des

\footnotetext{
9 Ambroise-Rendu 2013.
}

10 Nous tenons à remercier vivement l'équipe des Archives gaies du Québec pour son accueil et les conseils dont elle nous a fait bénéficier.

11 Cette circulation d'idées ne doit pas nous faire oublier que le Québec et le Canada forment deux espaces militants relativement distincts, comme le souligne Pascale Dufour à propos de mouvements militants plus contemporains (2013 : 37-38). 
mouvements gais), mais également à l'absence, au sein de ce corpus, des positions adoptées par les mouvements lesbiens. Cette absence a sans doute à voir avec ce qu'Anne Révillard nomme «l'invisibilité lesbienne ». Invisibilité, tout d'abord, dans l'espace du militantisme, en raison notamment de «l'articulation [...] conflictuelle entre féminisme et mouvement homosexuel », qui a traversé l'histoire des mouvements lesbiens ${ }^{12}$. Ainsi, pour la question qui nous concerne, les positions des femmes apparaissent d'abord comme les positions des mouvements féministes au sujet des violences sexuelles, condamnant les revendications pédophiles sur la sexualité des enfants. Invisibilité, ensuite, dans l'espace public, où les lieux de drague et de rencontre homosexuels sont, dans leur majorité, occupés par les gais. Or la cause de la majorité sexuelle s'est historiquement structurée autour des cas de répression dans l'espace public. Cette différence n'est pas sans effet sur la structuration de mouvements de contestation : "par opposition à la répression ouverte subie par les gays, [l'invisibilité lesbienne] rend plus difficile à formuler la position victimaire qui légitime habituellement l'action politique $»^{13}$. Si ces facteurs d'invisibilisation jouent sans doute sur nos sources, cela ne signifie pas que l'histoire des rapports entre majorité sexuelle et militantisme lesbien ne soit ni possible ni à faire. Mais nous n'avons pas, pour l'heure, les moyens de l'aborder.

Cet article est structuré en deux parties. Nous présentons, dans un premier temps, la manière dont se sont formées, dans les mouvements gais français et québécois des années 1970, les critiques du «double standard» en matière de majorité sexuelle, aboutissant, en certains cas, à des mises en cause radicales de l'idée même d'une majorité sexuelle. Dans un second temps, nous analysons les révisions législatives qui, dans les années 1980, en France et au Canada, ont répondu à ces revendications, dans un contexte de cristallisation de la nouvelle figure du danger : le pédophile.

12 Revillard 2002 : 175. Sur les tensions entre féminisme et lesbianisme au Québec depuis les années 1960 et 1970, voir notamment Lamoureux 1998, et Chamberland 2002.

13 Révillard 2002 : 176-177. 


\section{Les années 1970 : militantisme gai et sexualité des mineurs}

Au début des années 1970, la majorité sexuelle légale, en France et au Canada, présente une structure comparable. La loi fixe un âge général - 15 ans en France, 14 ans au Canada - et prévoit un régime spécial pour les relations homosexuelles -21 ans dans les deux cas. Dans les deux pays, les homosexuels sont stigmatisés, perçus comme une menace pour la jeunesse et sa moralité. En France, en 1960, l'Assemblée nationale vote l'amendement Mirguet, qui classe l'homosexualité comme « fléau social», en l'incluant dans une liste de " fléaux », notamment l'alcoolisme, la tuberculose et la prostitution. Cet amendement sera suivi, quelques mois plus tard, d'un doublement des peines encourues pour outrage public à la pudeur "lorsqu'il consistera en un acte contre nature avec un individu de même sexe ». Au Canada, en 1954, une réforme du Code criminel intègre les personnes condamnées pour "grossière indécence» à celles qui peuvent être classées comme souffrant de "psychopathie sexuelle », les rendant ainsi passibles, selon une loi de 1949, de détention à durée indéterminée ${ }^{14}$. Les premières mobilisations politiques gaies, au début des années 1970, s'attaquent à ces normes juridiques, aux représentations sociales qui les légitiment, et aux pratiques répressives qui en découlent.

\section{Des groupes d'expérimentation}

L'émergence de mouvements militants gais, en France et au Québec, suit une chronologie très proche. En France, le Front homosexuel d'action révolutionnaire (FHAR) est fondé au début de l'année 1971, par des féministes et des gais. S’il se réunit jusqu'en 1974, son activité s'est principalement concentrée entre 1971 et $1972^{15}$. Au Québec, la vie du Front de libération homosexuel (FLH) est plus brève ${ }^{16}$. Fondé en avril 1971 autour de la revue Mainmise, principal journal de la contre-culture $^{17}$, il se dissout à l'été 1972, à la suite d'une descente de police dans ses locaux. Ces premiers groupes se situent à la fois dans

\footnotetext{
14 Hurteau 1993 : 60.

15 Sibalis 2010.

16 Higgins 1996 et 1999.

17 Warren 2012.
} 
la proximité et le décalage avec d'autres groupes politiques radicaux, qu'il s'agisse, en France, des organisations trotskystes et maoïstes, ou au Québec, du mouvement indépendantiste et révolutionnaire structuré autour du Front de libération du Québec (FLQ). Dans les deux pays, les groupes homosexuels s'attaquent au virilisme qui traverse ces mouvements et à leur refus de faire des questions sexuelles des enjeux politiques. Sur une petite note manuscrite conservée aux Archives gaies du Québec, un militant du FLH, commentant le Manifeste du FLQ, reproche à ce dernier de traiter Pierre-Elliott Trudeau, alors Premier ministre du Canada, de «tapette». Cette volonté de renverser les formes habituelles de l'injure se retrouve, en France, dans le récit d'une manifestation publié par le journal militant L'Antinorm: "Les gauchistes crient leurs propres slogans anti-flic, et notamment "enculés". Moi j’ai gueulé avec les copains du FHAR: "Ah que c'est bon de se faire enculer" ${ }^{18}$. Ces premiers groupes cherchent à lier libération politique, lutte des classes et défense de l'homosexualité. Au Québec, un article de Mainmise, intitulé "L'homosexualité est-elle une arme politique?", souligne que "jamais la bourgeoisie n'a toléré la libre disposition d'un corps en face de n'importe quel autre, et en particulier chez les mineurs ${ }^{19}{ }^{9}$. En France, le FHAR, dans un article intitulé «Les pédés et la révolution», analyse «l'exploitation sexuelle » comme partie intégrante du fonctionnement capitaliste ${ }^{20}$.

Dans les prises de position de ces «homosexuels révolutionnaires $»^{21}$, la sexualité des mineurs, y compris des plus jeunes, est un élément important. En France, un Front de libération de la jeunesse est créé au sein du FHAR. Discutant de l'âge de la majorité sexuelle, il adopte comme slogan de manifestation: «les mineurs ont envie de se faire baiser $»^{22}$. Ces expérimentations sont moins prégnantes au Québec : le FLH réserve d'ailleurs ses adhésions aux personnes majeures. Dans ce contexte, la défense de la sexualité

\footnotetext{
18 L'Antinorm, n¹, décembre 1972-janvier 1973.

19 Mainmise, $\mathrm{n}^{\circ} 10$, janvier 1972.

20 Tout!, n¹2, 23 avril 1971. Repris dans FHAR 1971: 51 et sq.

21 Le Derff 1974 ; Hocquenghem 1972.

22 Girard 1981: 105.
} 
des mineurs s'exprime essentiellement dans des publications comme Mainmise $^{23}$. Une autre revue québécoise, Omnibus, relaye en 1973 les positions controversées de l'écrivain Jean Simoneau, qui se déclare ouvertement en «défense de la pédérastie » et de l'amour avec les « petits gars », à partir de 10-12 ans ${ }^{24}$.

Ces premiers mouvements gais partagent avec les mouvements d'extrême-gauche une volonté de rupture radicale, qu'ils ne conçoivent cependant pas dans la perspective de la prise du pouvoir. Ils s'apparentent, en cela, à des groupes d'expérimentation révolutionnaire dont la vitalité tient davantage à des pratiques collectives qu'à une structuration destinée à l'obtention de réformes politiques. En France, les réunions du FHAR se transforment progressivement en lieux de rencontres sans objectifs politiques explicites. À propos du Québec, Paul-François Sylvestre explique que "faute d'objectif précis, le FLH se dépolitise rapidement »25. Dans ce contexte, il ne se relève pas, en 1972, d'une descente musclée de la police dans ses locaux.

\section{La dénonciation des lois discriminatoires}

Dans la seconde moitié des années 1970, les mouvements gais précisent leurs revendications, qu'ils orientent vers la lutte contre la répression. Au Québec, et plus particulièrement à Montréal, l'année 1976 constitue, de ce point de vue, un moment crucial. À l'approche de l'organisation des Jeux Olympiques, la répression s'intensifie : les descentes policières dans les saunas et les bars gais se multiplient, jusqu'à l'arrestation de 89 personnes au Sauna Neptune, en mai 1976. Cet événement est le point de départ d'une restructuration du militantisme gai québécois: en mai 1976 est créé le Comité homosexuel anti-répression (CHAR), qui devient, quelques mois plus tard, l'Association pour les droits des gai(e)s du Québec (ADGQ). Dans un article publié en 1981, Ron Dayman, ancien coordinateur du CHAR, souligne que la création de l'ADGQ s'est accompagnée d'une marginalisation de l'idée d'une lutte de libération globale de tous les

\footnotetext{
23 Mainmise, $\mathrm{n}^{\circ}$ 6, septembre 1971.

24 Omnibus, vol. 2, n8, mars 1973.

25 Sylvestre 1979 : 53 .
} 
opprimés, au profit de la construction d'un «mouvement gai autonome basé sur la lutte démocratique des droits des gais ${ }^{26}$. Dès sa création, l'ADGQ définit sa première bataille : la modification de la Charte québécoise des droits et des libertés de la personne, promulguée en juin 1976 par le gouvernement libéral, qui ne fait pas mention de l'orientation sexuelle comme motif de discrimination. Il faut attendre une nouvelle rafle pour que des changements surviennent. Le 21 octobre 1977, la police arrête 144 hommes dans les bars Truxx et Le Mystique. En deux nuits, cet événement - décrit comme «le Stonewall du Québec $»^{27}$ - entraîne des manifestations spontanées et une importante médiatisation. Six jours plus tard, l'ADGQ présente un mémoire destiné à la modification de la Charte. Les réactions politiques s'enchaînent, jusque la réforme attendue, votée le 15 décembre 1977.

Le combat est cependant loin d'être terminé. En cause notamment, à un niveau fédéral cette fois, les articles 155 et 158 du bill Omnibus de 1969, qui fixent à 21 ans l'âge du consentement à la sodomie. Comme le souligne Ron Dayman, si « en principe, ces articles peuvent s'appliquer à des hétérosexuel(le)s [...], en pratique, les homosexuels (masculins) [sont] à peu près les seules victimes de ces lois archaïques $»^{28}$. Cette disposition parait d'autant plus inacceptable que depuis 1971, le seuil d'âge de la majorité civile, dont la fixation est de compétence provinciale, a été abaissé à 18 ans au Québec. Publié entre 1976 et 1977, Gay Montreal, «journal de grande envergure» pour le mouvement gai du milieu des années 197029, incarne la transition vers un militantisme recentré sur la cause gaie ${ }^{30}$. Le journal s'attache, en particulier, à déconstruire l'image de la «perversion» transmise par les relations homosexuelles avec des jeunes. Dans le même temps, il met régulièrement en garde ses

\footnotetext{
26 Le Berdache, n²0, mai 1981.

27 Sivry $1998: 243$.

28 Gay Montréal, n¹6, 2 novembre 1976.

29 Giguère 2012 : 1.

30 Publiant une chronique du livre de Jean Le Derff, Homolibre, défenseur d'une «révolution globale», le journal dit son désaccord avec l'idée qu'il faut déconstruire tous les pouvoirs, et fait valoir la crainte des excès révolutionnaires de 1971 (Gay Montréal, nº, 15 juin 1976).
} 
lecteurs face aux risques que comportent ces relations. Un jeune homme de 25 ans raconte ainsi, dans la rubrique "Courrier des lecteurs ", que son neveu de 13 ans dort dans sa chambre pendant l'été, et semble lui faire des avances. Le journal lui recommande la plus grande prudence en raison de son âge: son neveu pourrait déclencher un scandale ou exercer un chantage ${ }^{31}$. Le bill Omnibus de 1969 est accusé d'être une " arme à double tranchant », selon les mots de Paul-François Sylvestre dans Le Berdache, journal créé en 1979 sous l'impulsion de militants de l'ADGQ et de quelques transfuges de Mainmise: "Serait-ce qu'il faut plus de maturité pour s'aimer entre personnes du même sexe ? $»^{32}$

En France, un processus de conversion similaire s'opère au même moment. D’une articulation de la libération sexuelle et de la lutte des classes, les mouvements gais s'orientent vers une lutte spécifique contre la répression ${ }^{33}$. Un tract des Groupes de libération homosexuelle (GLH) fait ainsi mention, en 1978, d'un « contexte de tracasseries policières généralisées sur tous les lieux de rencontre homosexuels des grandes villes françaises ${ }^{34}$. C'est dans ce contexte qu'à l'été 1979, est fondé le Comité d'urgence anti-répression homosexuelle (CUARH). Cette « urgence» est notamment défendue par Claude Courouve et l'Association laïque pour l'étude du problème de l'homosexualité (ALEPH), fondée en 1975, pour dépasser «le clivage entre réformistes et révolutionnaires » et unir les militants autour de revendications législatives précises ${ }^{35}$. En novembre 1980, le premier numéro du journal du CUARH, Homophonies, publie une "lettre ouverte aux candidats à la présidentielle», exigeant, parmi d'autres revendications, l'abrogation de l'article 331-2 du Code pénal, responsable du «double standard» entre les âges du consentement homosexuel et hétérosexuel.

\footnotetext{
31 Gay Montréal, n², 4 mai 1976.

32 Le Berdache, $\mathrm{n}^{\circ} 3$, septembre 1979.

33 Bérard 2014a.

34 BNF, Tract, "Contre la répression des homosexuels », 11 octobre 1978, Groupes de libération homosexuelle (France), Recueil. Documents d'information, 1974.

35 Centre d'information ALEPH, Contre-nature? La répression judiciaire de l'homosexualité, 1978, brochure.
} 


\section{Pour une libération de la sexualité des mineurs}

Ces critiques qui, dans les années 1970, entourent les discriminations liées au consentement sexuel, se doublent de prises de position plus diffuses, mais aussi plus radicales, qui visent une libération sexuelle des mineurs, y compris des plus jeunes. Ces prises de position s'expriment, en particulier, dans différentes publications d'écrivains engagés dans une posture de défense de la sexualité avec des enfants, quand ils se disent pédophiles, ou avec des jeunes adolescents, quand ils se disent pédérastes. En France, l'écrivain Gabriel Matzneff, présenté en 1974, dans le Magażine littéraire ${ }^{36}$, comme "une figure importante de notre littérature », revendique sa «pédérastie» et fustige l'association de « l'amour des gosses » et de « l'idée de violence » ${ }^{37}$. En 1974 toujours, Tony Duvert, reconnu un an plus tôt pour avoir reçu le prix Médicis, signe un essai virulent, s'attaquant non seulement au vieux modèle de la "famille patriarcale ${ }^{38}$, mais également aux progressistes qui, selon lui, reconnaissent la sexualité des enfants sans pour autant admettre que celle-ci puisse s'exprimer à tout âge. Signe d'une circulation de ces prises de position, entre la France et le Québec, Duvert est invité à s'exprimer, en 1980, dans le cadre d'un «dossier pédérastie » publié dans Le Berdache. Rappelant son amour des adultes, quand il était enfant, et son amour des enfants, maintenant qu'il est adulte, il explique n'avoir été «qu'un gamin vicieux, un délinquant mineur et un adulte criminel ${ }^{39}$.

À la fin des années 1970, l'affaire de Jacques Dugué, et les réactions qu'elle suscite, illustrent la portée de ces prises de position, qui ont un temps réussi à prendre place dans l'espace public et médiatique français. Arrêté pour des relations sexuelles avec des mineurs, et suspecté de proxénétisme par le journal d'extrême-droite Minute, Dugué fait la une du quotidien France-Soir, qui évoque « une écœurante affaire de mœurs » où un «soi-disant éducateur aurait livré

\footnotetext{
36 Marc de Smedt, "Matzneff et la bisexualité », Le Magazine Littéraire, n94, $1^{\mathrm{er}}$ novembre 1974.

37 Matzneff $1974: 40$.

38 Duvert $1974: 17$

39 Tony Duvert, « Pédéraste, ou pédophile », Le Berdache, novembre 1980.
} 
à la prostitution internationale des régiments de gamins $»^{40}$. Le journal Gai-Pied, créé en 1979, dénonce « la coalition de la France profonde et de nos censeurs bureaucrates, anonymes et repus de cause contre les pervers $»^{41}$. À l'approche du procès qui condamne l'accusé à 6 ans de prison, le journal du CUARH Homophonies souligne qu'aucun enfant n'a été contraint et estime que «la loi se moque du consentement $»^{42}$. Cette affaire symbolise une période d'ouverture des possibilités d'expression publique de la pédophilie. Dans une tribune publiée par le quotidien Libération, quinze jours après l'article de France-Soir, Dugué revendique ainsi les actes qui lui sont reprochés et s'en prend, plus généralement, à la tendance à « considérer les enfants comme des demi-hommes, dépendants et irresponsables $»^{43}$.

Sans qu'elles n'aient disposé, au Québec, du relais médiatique dont elles ont profité en France, les revendications pédophiliques et pédérastiques ont néanmoins traversé le mouvement gai québécois. Dès sa création, Le Berdache, comme le fera d'ailleurs Gai-Pied côté français ${ }^{44}$, affiche sa solidarité avec la revue torontoise The Body Politic, engagée dans une série de procès suscités par la publication, dans son numéro de décembre 1977-janvier 1978, d’un article intitulé «Men Loving Boys Loving Men », rédigé par le journaliste et militant Gerald Hannon ${ }^{45}$. Dans le champ intellectuel québécois, l'écrivain Jean Simoneau est la figure la plus importante de ce courant. Dans le « dossier pédérastie » du Berdache, il rappelle les habitudes sexuelles de la Grèce antique, défendant la "valeur éducative» de ce qu'il nomme «l'amourajoie» : « je n'ai jamais connu de jeunes qui n'ont pas été plus heureux après nos rencontres, sauf un qui, à cause de la réaction de ses parents et du procès qu'ils exigèrent, a piqué une dépression $»^{46}$. La réaction sociale est accusée : le psychologue Alain Bouchard analyse, quelques pages plus loin, le «traumatisme de l'enfant», sommé «d'abandonner ses pratiques honteuses, vicieuses, monstrueuses qui

\footnotetext{
$40 \quad$ France-Soir, 13 janvier 1979.

41 Le Gai-Pied, n¹3, mai 1979.

42 Homophonies, n², décembre 1980.

43 Libération, 21 janvier 1979.

44 Gai-Pied, n², mai 1979; Gai-Pied, n90, octobre 1983.

45 Pour un regard sur la revue The Body Politic, voir Connors Jackman 2011.

46 Jean Simoneau, «De la "chûte des anges” », Le Berdache, novembre 1980.
} 
déplaisent à papa, maman et au petit Jésus $»^{47}$. Comme en France, l'ordre moral est dénoncé comme un ordre familial bourgeois, préservé par quelques "spécialistes" de l'enfance, des pédagogues aux psychiatres, participant, selon le poète et essayiste Paul Chamberland, cofondateur, en 1963, de la revue politique et culturelle Parti-Pris, d'« un vaste et complexe système de surveillance - familialisation, réclusion scolaire, pédagogie » ${ }^{48}$. Au Québec, sont également visés les acteurs catholiques, fragilisés en ces temps d'émancipation culturelle et politique de la société québécoise, et cherchant en retour, selon Simoneau, à préserver l'ordre et la tradition dans un «mariage de raison » avec «l'establishment anglophone [et] colonisateur ${ }^{49}$.

Dans les années 1970, les mouvements militants gais, en France et au Québec, suivent une trajectoire similaire, qui les conduit d'un moment d'émergence radical et expérimental à un temps de structuration d'organisations visant l'obtention de transformations législatives plus ciblées. C'est dans ce cadre qu'est posée la question du « double standard » de la majorité sexuelle, et, dans certains cas, celle de l'idée même de majorité sexuelle. Cette politisation de la question de la majorité sexuelle a conduit les législateurs des deux pays, dans les années 1980, à adopter un nouveau cadre légal.

\section{Les années 1980 : la majorité sexuelle réformée, et réaffirmée}

En France, comme au Québec et plus largement au Canada, l'âge du consentement homosexuel a été réformé, pour être abaissé, dans le courant des années 1980. Dans les deux pays, cette réforme s'est inscrite dans une redéfinition juridique plus générale des agressions sexuelles, faisant notamment suite aux mobilisations féministes destinées à obtenir la reconnaissance de la gravité et de l'ampleur des violences sexuelles ${ }^{50}$. Si les militantes féministes qui ont œuvré à cette reconnaissance n’ont pas ignoré le caractère discutable du seuil légal

\footnotetext{
47 Alain Bouchard, "Les dramaturges du sexe », Le Berdache, n¹5, novembre 1980.

48 Paul Chamberland, «Le massacre des innocents », Le Berdache, $\mathrm{n}^{\circ} 15$, novembre 1980.

49 Jean Simoneau, «La répression sexuelle des jeunes », Le Berdache, n 9, avril 1980.

50 Sur la France : Bérard 2014b ; sur le Québec, voir par exemple les textes militants rassemblées dans Dumont \& Toupin 2003 : 549-569.
} 
de la majorité sexuelle, elles ont largement récusé l'idée, portée par les pédophiles, qu'il était possible de se passer de toute notion de majorité sexuelle. Dans son ouvrage Against Our Will: Men, Women and Rape, paru en 1975, Susan Brownmiller estime ainsi que la fixation d'un âge est pour une part arbitraire, mais que la définition d'une telle limite - elle mentionne l'âge de 12 ans - est un compromis nécessaire pour protéger les enfants contre les agressions sexuelles ${ }^{51}$.

La transformation juridique des règles du consentement sexuel a pris des chemins sensiblement différents dans les deux pays. Si la discrimination légale sur la majorité sexuelle a, en France, été abolie dès 1982, il a fallu attendre 1988 pour qu'au Canada, elle soit seulement atténuée... avant d'être définitivement abrogée, au Québec, en 1998.

\section{En France : vers l'abrogation de la loi discriminatoire}

En France, à la fin des années 1970, les revendications des mouvements gais atteignent la sphère des partis politiques et des enceintes législatives. Le programme commun entre communistes et socialistes, publié en 1978, reprend plusieurs des revendications militantes. Celles-ci trouvent également un écho par l'intermédiaire du sénateur radical Henri Caillavet. Ce parlementaire, qui a été rapporteur des lois sur l'avortement et le divorce par consentement mutuel, dépose en février 1978 une proposition de loi visant, entre autres, à dépénaliser les relations homosexuelles avec les mineurs de plus de quinze ans. En juin 1978, lors de la discussion de la loi sur le viol obtenue par les mobilisations féministes, le Sénat adopte un amendement gouvernemental qui reprend, en partie, le projet Caillavet. Mais en avril 1980, le député conservateur Jean Foyer, adversaire le plus tenace des mouvements homosexuels, obtient le rétablissement de l'article controversé. La mobilisation qui en résulte n'y change rien: le Sénat "fait volte-face ${ }^{52}$, et refuse finalement d'abroger l'article. Les députés socialistes se saisissent du Conseil constitutionnel, qui en décembre 1980, rejette leur recours ${ }^{53}$. Seule victoire : est votée l'abrogation de l'article du Code pénal qui, suite à

\footnotetext{
51 Brownmiller 1975.

52 Le Gai-Pied, n²0, novembre 1980.

53 Conseil constitutionnel, Décision nº8-125 du 19 décembre 1980.
} 
l'amendement Mirguet, doublait la peine minimum pour outrage public à la pudeur dans le cas de rapports homosexuels.

Dans la perspective des élections présidentielle de 1981, le CUARH se tourne vers les candidats. Si tous les candidats de gauche se montrent favorables à leurs demandes, Jacques Chirac et Valéry Giscard d'Estaing ne répondent pas ${ }^{54}$. Suite à l'élection de François Mitterrand, Gai-Pied titre "sept ans de bonheur » ${ }^{55}$. La loi d'amnistie votée par la nouvelle majorité porte, entre autres, sur les condamnés pour homosexualité avec des mineurs de plus de 15 ans ${ }^{56}$. Le 6 août 1981, 156 personnes détenues pour ce motif sont libérées. La navette législative de la loi d'abrogation se met en route dans l'année 1981. Suite au premier vote de l'Assemblée, en janvier 1982, Homophonies titre «l'homosexualité n'est plus un délit», et "Vive les homos de 15 ans ! $»^{57}$. Le 27 juillet 1982, la loi sur l'abrogation est adoptée. GaiPied peut annoncer : «Il a 15 ans, il est libre » ${ }^{58}$.

Une partie des militants refuse de considérer cette victoire comme une fin en soi. Dénonçant à la fois les attaques de la droite, et la frilosité du pouvoir, Gai-Pied pointe ainsi «les scories de l'abrogation », car, explique-t-il, «il est fort probable que cette mesure libérale, qui n'est jamais que la disparition d'une inégalité scandaleuse, devienne en fait la barrière d'enfermement de la pédophilie, si ce n'est déjà fait » ${ }^{59}$. Cette volonté d'aller plus loin est relayée conjointement par le CUARH et les militants regroupés au sein du Groupe de recherche pour une enfance différente (GRED). Le premier numéro de son bulletin, Le petit gredin, dénonce, entre autres, la pénalisation des relations avec des mineurs de moins de quinze ans ${ }^{60}$. Si en avril 1982, Simone Iff, conseillère au ministère des Droits de la femme, déclare que la réflexion du gouvernement n'est pas close, elle indique cependant qu'il ne s'agit

\footnotetext{
54 Homophonies, $\mathrm{n}^{\circ}$ 6, avril 1981.

55 Le Gai-Pied, n²7, juin 1981.

56 La criminalité en France d'après les statistiques de la police judiciaire, ministère de l'Intérieur, DGPN, Service central d'étude de la délinquance, 1981.

57 Homophonies, $\mathrm{n}^{\circ} 15$, janvier 1982.

58 Le Gai-Pied, n ${ }^{\circ}$, septembre 1982. Sur ce point, voir aussi Idier 2013.

59 Le Gai-Pied, $\mathrm{n}^{\circ} 34$, janvier 1982.

60 Le petit gredin, journal du Groupe de recherches pour une enfance différente, $\mathrm{n}^{\circ} 0,1981$.
} 
pas de la priorité du gouvernement ${ }^{61}$. Robert Badinter, garde des Sceaux, et Gisèle Halimi, militante féministe devenue députée socialiste, réaffirment quant à eux, et plus directement, l'utilité de l'arsenal judiciaire concernant la majorité sexuelle.

En octobre 1982, l'affaire judiciaire dite du «Coral» éclate, refermant les possibilités d'expression publique de la pédophilie, qui s'étaient - brièvement - ouvertes à la fin des années 1970. Le «Coral» était un lieu symbolique des pratiques des « années 68 », qui accueillait, depuis le milieu des années 1970, des personnes en grande difficulté, dont des enfants et des adolescents, avec l'objectif de les aider sans recourir aux pratiques disciplinaires des foyers et institutions traditionnels. Le quotidien France-Soir publie des révélations, au demeurant fort imprécises, sur les pratiques sexuelles supposées des animateurs du centre : on passe du viol des enfants à leur disparition, jusqu'à l'organisation d'un réseau d'exploitation sexuelle impliquant des membres de cabinets ministériels socialistes. Si l'affaire est vite ramenée à des proportions moins sensationnelles, elle marque néanmoins une distance nouvelle entre le pouvoir socialiste et les mouvements gais. Dénonçant les «rumeurs » et l'emballement de la presse, Gai-Pied décrit «l'embarras terrifié du pouvoir face à cette affaire $»^{62}$. En janvier 1983, pour Homophonies, les quelques espoirs d'une réforme générale de la majorité sexuelle paraissent déjà bien loin ${ }^{63}$. Gai-Pied, en novembre 1982, explique que "dans un rapport avec un adulte, le libre choix de l'enfant est loin d'être aussi évident que l'affirment les théoriciens pédérastes ${ }^{64}$. Le débat se clôt progressivement, la gauche y étant d'autant plus incitée que la droite fait de la pédophilie l'un des vecteurs de médiatisation de la question de l'insécurité. Celle-ci tend alors à devenir, comme le déplore Tony Duvert en 1982, « le Crime par excellence » ${ }^{65}$.

Les défenseurs de la pédophilie sont de plus en plus isolés. Ils le sont également vis-à-vis des militantes féministes qui condamnent la

\footnotetext{
61 Le Gai-Pied, n³7, avril 1982.

62 Gai-Pied Hebdo, n 45, 27 novembre 1982.

63 Homophonies, $\mathrm{n}^{\circ} 27$, janvier 1983.

64 Gai-Pied Hebdo, n45, 27 novembre 1982.

65 Tony Duvert, «L'inceste ou la prison », Gai-Pied, n³9, juin 1982.
} 
pédophilie au nom de la dénonciation plus générale des violences sexuelles. Ainsi Leila Sébbar s'en prend-elle aux pédophiles qui, comme les "père, médecin, pédagogue, curé, éducateur, psy, juge, flic, commissaire politique », soustraient le corps de l'enfant à sa mère ${ }^{66}$.

\section{Au Québec et Canada : priorité à la protection de la jeunesse}

Comme en France, la question de la majorité sexuelle, au Canada, est intimement articulée à la redéfinition des agressions sexuelles. Prolongement d'un Rapport sur les infractions sexuelles publié par la commission de réforme du droit du Canada en 1978, le bill C-53, déposé en janvier 1981, précise les interdits sexuels à l'égard des mineurs. Inspiré par les mobilisations féministes, il définit plus généralement trois niveaux d'agressions sexuelles, suivant leur degré de violence, pour élargir la définition du «viol» et mettre fin à l'exclusion des viols entre époux. Le projet de loi propose également une réforme de l'article 158 du Code criminel, en abaissant, de 21 à 18 ans, l'âge du consentement pour les actes de "sodomie » et de "grossière indécence». Salué comme un progrès ${ }^{67}$, bien qu'il maintienne de fait un écart discriminant, ce projet de loi ne sera finalement jamais adopté. Il est remplacé par le bill C-127, adopté en octobre 1982. Des dispositions présentées plus haut, seule est retenue la redéfinition, en trois niveaux, des agressions sexuelles. Si le projet de loi est une victoire pour les mouvements féministes, les mouvements gais, de leur côté, s'indignent de ce revirement, qu'ils perçoivent comme le signe d'un épuisement de leurs revendications. Un article du premier numéro de Sortie, journal créé par quelques rédacteurs du Berdache, qui cesse alors de paraitre, accuse notamment le conservatisme de «la droite style "moral majority" » ${ }^{68}$. Les positions les plus radicales, pédérastiques voire pédophiliques, sont de plus en plus contestées. Des militantes féministes se font entendre, faisant valoir que ces positions sont toujours défendues du point de

\footnotetext{
66 Sebbar $1980: 15$.

67 Le Berdache, février 1981.

68 Sortie, nº1, p. 14.
} 
vue des adultes, et occultent le caractère fondamentalement inégal des relations entre adultes et enfants ${ }^{69}$.

À y regarder de plus près, plusieurs facteurs peuvent rendre compte de l'échec du bill C-53. D’une part, les militants québécois et canadiens ne disposent pas, au début des années 1980, de relais - politiques et médiatiques - aussi influents que leurs homologues français. D'autre part, là où, en France, la question de la majorité sexuelle est directement liée à celle de la (dé)pénalisation de l'homosexualité, au Canada, l'article 158 ne se réfère formellement qu'à des pratiques sexuelles (sodomie, grossière indécence), et non pas à une orientation sexuelle. Dans ce cadre, les débats sur la réforme de l'article 158 sont inscrits dans des discussions plus générales relatives à la protection de la jeunesse, objet de multiples controverses au début des années 1980. Le juriste Ian Wallace voit d'ailleurs dans ces tensions et controverses la raison principale de l'échec du bill C-53 : pour éviter de retarder l'ensemble du processus législatif, les principales organisations féministes canadiennes, particulièrement influentes à Ottawa, obtiennent de ne retenir, dans le bill C-127, que ce qui compte le plus à leurs yeux : la redéfinition du viol ${ }^{70}$.

Il faut attendre la publication, en 1984, des conclusions d'un Comité présidé par le professeur Robin Badgley, chargé d'une étude sur les infractions sexuelles commises à l'égard des mineurs, pour que soit reposée la question des seuils de la majorité sexuelle. Dans son rapport, le Comité recommande la condamnation: de tout « attouchement sexuel » avec un enfant - garçon ou fille - de moins de 14 ans; des "rapports sexuels vaginaux » pour les jeunes filles de moins de 16 ans; des "actes de sodomie » pour toute personne des deux sexes de moins de 18 ans $^{71}$. Cherchant à écarter toute accusation de discrimination, le Comité précise que la sodomie concerne aussi bien des comportements homosexuels qu'hétérosexuels. Pourtant,

69 Ces critiques sont par exemple exposées dans un article de The Body Politic qui revient sur les objections adressées au texte de Gerald Hannon « Men loving boys loving men » : Bearchell, Bebout \& Wilson 1982.

70 Wallace 1988.

71 Badgley $1984: 23$. 
quelques lignes plus loin, il dévoile ses présupposés en évoquant explicitement « l'orientation sexuelle» :

Une grande partie du corps médical s'accorde à dire que l'orientation sexuelle est déjà fixée à partir de 16 ans. Mais il existe des avis contraire [...] Le Comité n'abaisserait donc pas l'âge de la liberté sexuelle à 16 ans en l'absence de preuves indiscutables qu'une telle réduction n'entrainerait aucun risque dans le développement du comportement sexuel ${ }^{72}$.

Après une tentative avortée, en 1986, ces recommandations sont partiellement adoptées, le 1er janvier 1988, dans le cadre du bill C-15. La loi abolit la distinction entre jeunes garçons et jeunes filles et fixe un âge général du consentement à 14 ans. Elle abroge la qualification de "grossière indécence», mais pas la fixation d'un âge spécifique pour le consentement à la sodomie, qu'elle abaisse cependant de 21 à 18 ans. Alors que sept ans auparavant, le bill C-53 avait provoqué de nombreuses réactions, relayées au sein du Berdache ainsi que des premiers numéros du magazine Sortie, ce dernier, en 1987 et 1988, ne fait aucune mention du processus législatif qui aboutit à l'adoption du bill C-15. À l'époque, ce désintérêt était relativement récent. À l'approche des élections fédérales de 1984, l'ADGQ interpellait encore, dans Le Petit Berdache, réminiscence éphémère du Berdache, les différents partis politiques pour connaitre leurs positions sur l'âge du consentement.

Au début des années 1990, c'est autour d'une affaire judiciaire que la question se pose à nouveau. Il s'agit, au niveau québécois, du procès d'Henry Roy, condamné une première fois par la cour du Québec, en octobre 1994, pour avoir entretenu, quatre ans auparavant, des relations sexuelles anales avec une personne mineure. Suite à l'appel d'Henry Roy, la Cour d'appel du Québec, le 15 avril 1998, révise le premier jugement et déclare inconstitutionnel l'article 159 du Code criminel, qui « empêche les homosexuels mineurs d'avoir des activités sexuelles habituelles, consensuelles et dans l'intimité, avant l'âge de 18 ans $»^{73}$. Seize ans après la France, le «double standard » est ainsi abrogé.

\footnotetext{
72 Badgley $1984: 21$.

73 R.c. Roy, 1998, 12775 (QC CA).
} 


\section{L'ordre sexuel redéfini : les familles sous surveillance}

De ces parcours législatifs, émerge une similitude frappante entre la France et le Canada : la redéfinition juridique de la majorité sexuelle s'est opérée parallèlement à la redéfinition juridique du viol. La discussion conjointe de ces deux enjeux, entremêlée puis démêlée, en fait les deux faces d'une même pièce: la redéfinition d'un ordre sexuel par la sortie des catégories héritées du XIX siècle. Avant ces réformes, la définition du viol comme relation non consentie et illicite garantissait l'impunité des violences commises dans le cadre conjugal. La définition de la majorité sexuelle, quant à elle, délimitait les frontières de l'ordre familial en protégeant la chasteté des jeunes filles, et la moralité (hétérosexuelle) des jeunes garçons. Dans le courant des années 1970, cet ordre familial est attaqué par les mouvements militants engagés dans une défense des sexualités opprimées : ceux-ci revendiquent et obtiennent que la loi définisse les violences sexuelles sans référence au statut marital, et la majorité sexuelle sans référence à l'orientation sexuelle.

Cette redéfinition de l'ordre sexuel, dans les années 1980, s'est opérée dans un moment d'épuisement des mouvements gais des années 1970, entrés dans les « années SIDA », qui reconfigurent leurs registres d'action et leurs revendications ${ }^{74}$. Leurs positions les plus radicales, liées notamment à la libération sexuelle des jeunes adolescents, voire des enfants, sont radicalement délégitimées, suscitant le scepticisme d'une part croissante des homosexuels euxmêmes. Les années 1980 et 1990 sont marquées, au contraire, par l'émergence d'une focalisation médiatique sans précédent sur la nouvelle figure du "pédophile», prédateur extérieur à l'espace familial, idéal-type du "stranger danger». Dans ses travaux sur les déviances sexuelles en Ontario (Canada), Elise Chenier analyse ainsi la cristallisation de cette nouvelle figure, qui reprend les traits caractéristiques du «psychopathe sexuel » des années 1930, appliqués à «l'homosexuel» dans les années 1950 et 1960 : développement arrêté, immaturité, désir incontrôlable et insatiable, influence

74 Pierre Chéhadé, "Mais où sont donc passés les militants des années 1970 ? ", Sortie, n51, septembre 1987, p. 14 ; Gai-Pied Hebdo, n 94,19 novembre 1983. 
corruptrice, etc. ${ }^{75}$. Cette représentation du «stranger danger», qui structure également le cadrage politique et médiatique de la pédophilie en France ${ }^{76}$ et au Québec ${ }^{77}$, se confronte cependant à la réalité ordinaire de ces violences sexuelles, mises en évidence par les enquêtes et prises en charge par les institutions de protection de la jeunesse : d'un côté comme de l'autre de l'Atlantique, les abus sexuels sur mineurs sont de fait, dans leur majorité, intrafamiliaux.

Dans les années 1980 et 1990, de manière plus institutionnelle, le problème des maltraitances intrafamiliales, et parmi elles celui des abus sexuels intrafamiliaux, ont ainsi impulsé, en France comme au Canada, une restructuration des politiques de protection de la jeunesse. Une note de synthèse du rapport Badgley, publié en novembre 1984 par le Service de recherche du gouvernement du Canada, soulignait déjà que «la majorité des [abus sexuels sur mineurs] sont commis par des personnes déjà connues des victimes (parents ou connaissances) et souvent dans la demeure de la victime ou à proximité $»^{78}$. Que l'on pense, en France, à la sophistication croissante de la politique d'Aide sociale à l'enfance, départementalisée en 1984, ou que l'on pense, au Québec, à la réorganisation progressive des institutions de protection de la jeunesse, jusqu'à la création, au début des années 1990, d'un réseau institutionnel unifié des Centres jeunesse, le maillage institutionnel de la «police des familles $\gg^{79}$ s'est ainsi à la fois étendu et resserré. Ses objectifs, quant à eux, se sont recentrés autour du thème de la "prévention des risques ", voire de la "prédiction des risques ", souvent familiaux, susceptibles d'entraver, sinon de menacer, le «bon développement» des enfants et des adolescents.

\footnotetext{
75 Chenier 2008.

76 Boussaguet 2008.

77 Pour un regard sur le traitement des abus sexuels des enfants par le quotidien québécois La Presse, voir Chamberland-Lajoie 2012.

78 Macdonald $1984: 3$.

79 Selon l'expression de Donzelot 1977.
} 
Dans les années 1970, les mouvements militants gais français et québécois entrent en lutte contre des lois aux formulations différentes. Mais leur histoire suit, durant une décennie, un parallélisme frappant. Fondés en lien avec des mouvements politiques révolutionnaires, les mouvements gais critiquent leur refus de prendre en compte les dimensions sexuelles de la contestation. Ils partagent, durant leurs premières années, une orientation radicale qui fait de la liberté sexuelle des mineurs, y compris les plus jeunes, l'enjeu de pratiques destinées à subvertir l'ordre économique, social et patriarcal. Au milieu des années 1970, le déclin du militantisme radical entraine la restructuration des espaces militants autour de revendications ciblées contre la répression dont les homosexuels sont victimes. Dans ce cadre, les militants dénoncent la discrimination dont ils sont victimes concernant l'âge de la majorité sexuelle. Leurs revendications sont reconnues comme légitimes et des processus de réformes sont initiés.

Ces processus sont assez différents. En France, l'alternance de 1981 entraîne le vote d'une réforme qui demeure comme le moment symbolique de dépénalisation de l'homosexualité. Au Canada, une réforme partielle n'est obtenue qu'en 1988, et des tribunaux québécois achèvent de discréditer la répression des "relations anales » avec des mineurs de plus de 14 ans en $1998^{80}$. Le point commun de ces réformes est de prendre place dans un contexte politique profondément transformé. D'un côté, l'existence d'une majorité sexuelle spécifiquement liée à une orientation ou des pratiques sexuelles perd toute légitimité. De l'autre, les discours des années 1970 qui visaient une libération radicale de la sexualité avec les enfants et les jeunes adolescents sont totalement disqualifiés. Dans un contexte où les mouvements féministes travaillent à la redéfinition - et à l'élargissement - du périmètre des violences sexuelles, la fixation d'une limite d'âge, fut-elle arbitraire, est considérée comme un compromis nécessaire. Les lois des années 1980, qui redéfinissent le viol et la majorité sexuelle, instituent un nouveau partage du permis et de l'interdit en matière sexuelle. Par l'affirmation d'une norme générale du

80 À noter qu'au Canada, l'âge de la majorité sexuelle générale a été l'objet, en 2008, dans le cadre d'une Loi sur la lutte contre les crimes violents du gouvernement conservateur, passant de 14 ans à 16 ans. 
consentement, elles éliminent les références aux statuts des personnes, à leur sexe et à leur orientation sexuelle. Dans le même temps, par la réaffirmation d'une norme indépassable de la majorité sexuelle, elles disqualifient toute relation sexuelle en deçà d'un certain âge. Cette reconfiguration normative ne saurait être interprétée en opposant la libération des années 1970 et le retour à l'ordre moral des années 1980. Les lois pénales comme les représentations médiatiques de la sexualité des plus jeunes sont certes marquées par les «paniques morales» liées à la pédophilie. Mais la définition de la majorité sexuelle et l'idée selon laquelle « la prime adolescence devrait être sans vie sexuelle ${ }^{81}$ s'inscrit $^{\prime}$ dans le temps plus long d'une transformation des âges de la vie. Dans un contexte de généralisation de la scolarité secondaire, l'enfance et la jeune adolescence sont, en effet, de plus en plus définies « comme une période où l'on étudie et où l'on vit en famille ${ }^{82}$.

\section{Bibliographie}

Ambroise-Rendu Anne-Claude, 2013, «Contre la dynamique incestueuse des familles : les intellectuels au secours de la pédophilie. Le temps des années 1970 », in Dorothée Dussy (dir.), L'Inceste, bilan des savoirs, Marseille, Éditions la Discussion, p. 19-38.

ARTIÈRes Philippe \& Michelle ZANCARINI-Fournel (dir.), 2008, 68, une bistoire collective (1962-1981), Paris, La Découverte.

BADGLEy Robin (dir.), 1984, Rapport du Comité sur les infractions sexuelles à l'égard des enfants et des jeunes. Recommandations, Ottawa, Gouvernement du Canada.

BÉRARD Jean, 2014a, «De la libération des enfants à la violence des pédophiles. La sexualité des mineurs dans les discours politiques des années 1970 », Genre, sexualité, société, 11 [En ligne : http://gss.revues.org/3134].

—, 2014b, «Dénoncer et (ne pas) punir les violences sexuelles. Luttes féministes et critiques de la répression en France, de mai 68 au début des années 1980 », Politix, 3/107, p. 61-84.

Boussaguet Laurie, 2008, La Pédophilie, problème public, France, Belgique, Angleterre, Paris, Dalloz.

Bozon Michel 2009, "Les âges de la sexualité. Entretien avec Marc Bessin», Mouvements, 59/3, p. 123-132.

81 Bozon 2009 : 129-130.

82 Bozon 2009 : 129. 
Brownmiller Susan, 1975, Against Our Will: men, women and rape, New-York, Simon $\&$ Chuster.

Chamberland Line, 2002, «La place des lesbiennes dans le mouvement des femmes », Labrys, études féministes, 1-2.

[En ligne : http://www.labrys.net.br/labrys1_2/chaberland2.html].

ChamberLand-Lajoie Jules, 2012, « Analyzing the portrayal of child sexual abuse in La Presse: the shifting dialectics of silence and denunciation ", master thesis in communication studies, Montreal, University of Concordia.

Chenier Elise, 2008, Strangers in our Midst: sexual deviancy in Postwar Ontario, Toronto, University of Toronto Press.

CONNORS JACKMAN Michael, 2011, "The body politic: at the genesis of sexual liberation in Canada ", Xtra Ottawa: Ottawa's Gay and Lesbian News, 236.

[http://www.dailyxtra.com/the-body-politic-78210]

CORriveau Patrice, 2006, La Répression des homosexuels au Québec et en France, Québec, Éditions du Septentrion.

Donzelot Jacques, 1977, La Police des familles, Paris, Éditions de Minuit.

Dumont Micheline \& Louise Toupin, 2003, La Pensée féministe au Québec, anthologie 1900-1985, Montréal, les Éditions du remue-ménage.

Dufour Pascale, 2013, Trois espaces de protestation: France, Canada, Québec, Montréal, Presses de l'Université de Montréal.

Duvert Tony, 1974, Le Bon Sexe illustré, Paris, Éditions de Minuit.

—, 1980, L'Enfant au masculin, Paris, Éditions de Minuit.

Foucault Michel, 1999, Les Anormaux, cours an Collège de France 1974-1975, Paris, Gallimard/Le Seuil.

FRONT HOMOSEXUEL D'ACTION RÉVOLUTIONNAIRE, 1971, Rapport contre la normalité, Paris, Éditions Champ libre.

GiguÈRE Nicolas, 2012, «Bref historique des archives gaies du Québec », L'Archigai, bulletin des archives gaies du Québec, 22, p. 1-2.

GiRARD Jacques, 1981, Le Mouvement homosexuel en France, 1945-1980, Paris, Syros.

Higgins Ross, 1996, "Vers la liberté : 25 années de militantisme au Québec», L'Archigai, bulletin des archives gaies du Québec, 5, p. 1-15.

—, 1999, De la clandestinité à l'affirmation, Pour une bistoire de la communauté gaie montréalaise, Montréal, Comeau et Nadeau.

HoCQuenghem Guy, 1972, Le Désir homosexuel, Paris, Éditions universitaires.

Hurteau Pierre, 1993, «L'homosexualité masculine et les discours sur le sexe en contexte montréalais de la fin du 19 e siècle à la Révolution tranquille », Histoire sociale/Social History, 26/51, p. 44-66. 
IDIER Antoine, 2013, Les Alinéas au placard: l'abrogation du délit d'bomosexualité (19771982), Paris, Cartouche.

LAMOUREuX Diane, 1998, «La question lesbienne dans le féminisme montréalais : un chassé-croisé », in Irène Demczuk \& Frank Remiggi (dir.), Sortir de l'ombre. Histoires des communautés lesbienne et gaie de Montréal, Montréal, VLB Éditeur, p. 167-185.

LE DERFF Jean, 1974, Homolibre, Montréal, Ferron Éditeur.

MACDONALD Donald, 1984, Les Infractions sexuelles à l'égard des enfants : le rapport Badgley, Ottawa, Service de recherche du gouvernement du Canada.

MARCHANT Alexandre, 2006, «Daniel Guérin et le discours militant sur l'homosexualité masculine en France (années 1950-années 1980) », Revue d'bistoire moderne et contemporaine, 53/4, p. 175-190.

MAtZnefF Gabriel, 1974, Les Moins de seize ans, Paris, Julliard.

Memmi Dominique, 2008, "Mai 68 ou la crise de la domination rapprochée », in Dominique Damamme, Boris Gobille, Frédérique Matonti \& Bernard Pudal (dir.), Mai-juin 68, Paris, Éditions de l'atelier, p. 35-46.

Revillard Anne, 2002, "L'identité lesbienne entre nature et construction », Revue du MAUSS, 1/19, p. 168-182.

SEBbar Leila, 1980, Le Pédophile et la maman : l'amour des enfants, Paris, Stock.

SIBALIS Michael, 2010, "L'arrivée de la libération gay en France. Le Front Homosexuel d'Action Révolutionnaire (FHAR)», Genre, sexualité \& société, 3 [En ligne : http://gss.revues.org/1428].

SimoneAu Jean, 1978, "Aimer les petits gars, féérie du monde adulte », in ColleCtIF (dir.), Sortir, Montréal, Éditions de l'Aurore, p. 139-155.

SIVRY Jean-Michel, 1998, "Traces militantes éphémères : l'ADGQ et Le Berdache », in Irène Demczuk \& Frank Remiggi (dir.), Sortir de l'ombre. Histoires des communautés lesbienne et gaie de Montréal, Montréal, VLB Éditeur, p. 235-263.

SylVESTRE Paul-François, 1979, Les Homosexuels s'organisent, au Québec et ailleurs, Ottawa, Éditions Homeureux.

Tamagne Florence, 2000, Histoire de l'bomosexualité en Europe, Berlin, Londres, Paris, 1919-1939, Paris, Le Seuil.

TréPANier Jean, 1986, «La justice des mineurs au Québec: 25 ans de transformations (1960-1985)», Criminologie, 19/1, p. 189-213.

WALLACE Ian, 1988, «Interest groups and the process of legislative reform Bill C-15 - A case study », Queens Law Journal, 13, p. 159-207.

WARrREN Jean-Philippe, 2008, Une Douce anarchie : les années 68 au Québec, Montréal, Boréal.

—, 2012, "Fondation et production de la revue Mainmise (1970-1978) », Mémoires du livre/Studies in Book Culture, 4/1 [En ligne: http://id.erudit.org/iderudit/1013326ar]. 